\title{
Multiuser Scheduling on the Downlink of an LTE Cellular System
}

\author{
Raymond Kwan, ${ }^{1}$ Cyril Leung, ${ }^{2}$ and Jie Zhang ${ }^{1}$ \\ ${ }^{1}$ Centre for Wireless Network Design, University of Bedfordshire, Park Square, Luton, LU1 3JU, UK \\ ${ }^{2}$ Department of Electrical and Computer Engineering, University of British Columbia, Vancouver, Canada V6T $1 Z 4$
}

Correspondence should be addressed to Raymond Kwan, raymond.y.c.kwan@gmail.com

Received 14 April 2008; Accepted 27 May 2008

Recommended by Ibrahim Develi

The challenge of scheduling user transmissions on the downlink of a long-term evolution (LTE) cellular communication system is addressed. In particular, a novel optimalmultiuser scheduler is proposed. Numerical results show that the system performance improves with increasing correlation among OFDMA subcarriers. It is found that only a limited amount of feedback information is needed to achieve relatively good performance. A suboptimal reduced-complexity scheduler is also proposed and shown to provide good performance. The suboptimal scheme is especially attractive when the number of users is large, in which case the complexity of the optimal scheme is high.

Copyright ( $\odot 2008$ Raymond Kwan et al. This is an open access article distributed under the Creative Commons Attribution License, which permits unrestricted use, distribution, and reproduction in any medium, provided the original work is properly cited.

\section{Introduction}

Orthogonal frequency division multiplexing (OFDM) is a multicarrier modulation technique that has been adopted in a variety of modern communication systems such as the digital subscriber lines (DSLs), wireless local area networks (WLANs), the Worldwide Interoperability for Microwave Access (WiMAX) [1], and long-term evolution (LTE) cellular networks. In order to exploit multiuser diversity and to increase flexibility in scheduling, orthogonal frequency division multiple access (OFDMA), in which multiple users can simultaneously share the subcarriers, is employed. The problem of power and subcarrier allocation in OFDMA systems has been the subject of much research (see [2, 3], and references therein).

In practice, due to limited signalling resources, subcarriers are allocated collectively. For example, on the downlink in LTE, subcarriers are grouped into resource blocks (RBs) of 12 adjacent subcarriers with an intersubcarrier spacing of $15 \mathrm{kHz}[4,5]$. Each RB has a time slot duration of 0.5 milliseconds, which corresponds to 6 or 7 OFDM symbols. (The actual value depends on whether an extended or normal cyclic prefix is used.) The smallest resource unit that a scheduler can assign to a user is a scheduling block (SB), which consists of two consecutive RBs, spanning a subframe time duration of 1 millisecond $[4,5]$. The main issue to be addressed is how SBs are to be allocated to users, given that the channel qualities for the set of SBs associated with each user are different. Some studies on LTE-related scheduling have been reported in $[6,7]$ and the references therein.

One constraint in LTE downlink scheduling is that all SBs belonging to a single user can be assigned to only one modulation and coding scheme (MCS) in each transmission time interval (TTI) or scheduling period [4, page 326]. (This applies in the non-multiple-input-multiple-output (MIMO) configuration. For the MIMO configuration, a maximum of two different MCSs can be used for data belonging to two different transport blocks.) The durations of a TTI and $\mathrm{SB}$ are equal. To the best of our knowledge, the impact of this restriction on LTE scheduling has not been previously studied. In this paper, the challenging problem of multiuser scheduling is examined, taking into account this restriction.

\section{System Model}

In the time domain, each SB consists of a number, $N_{\mathrm{sb}}$, of OFDM symbols. Let $L$ be the total number of subcarriers and $L_{d}(\nu) \leq L$ be the number of data-carrying subcarriers for symbol $v$, where $v=1,2, \ldots, N_{\mathrm{sb}}$. Also, let $R_{j}^{(c)}$ be the code rate associated with the MCS $j \in\{1,2, \ldots, J\}, M_{j}$ be the constellation size of the MCS $j$ and $T_{s}$ be the OFDM symbol duration. Then, the bit rate, $r_{j}$, that corresponds to a single 
$\mathrm{SB}$ is given by

$$
r_{j}=\frac{R_{j}^{(c)} \log _{2}\left(M_{j}\right)}{T_{s} N_{\mathrm{sb}}} \sum_{\nu=1}^{N_{\mathrm{sb}}} L_{d}(\nu) .
$$

Let $U$ be the number of simultaneous users, and $N_{\text {tot }}$ be the total number of SBs that are available during each TTI. In addition, let $\mathcal{N}_{i}$ be a subset of the $N_{\text {tot }}$ SBs whose channel quality indicator (CQI) values are to be reported by user $i$; the size of $\mathcal{N}_{i}$ is denoted by $N_{i}$. It is assumed that the $N_{i}$ highest SB CQI values are fed back. Such a limited feedback scheme requires a smaller bandwidth albeit at the cost of a degraded system performance. We also assume that the total available power is shared equally among the users. As noted in $[8,9]$, the throughput degradation resulting from such an assumption is small when adaptive modulation and coding (AMC) is used, as is the case in LTE.

Let $\mathbf{x}_{i, n}, n=1,2, \ldots, N_{i}$ be a real scalar or vector reported (via a feedback channel) by user $i$ to indicate the collective channel qualities of all the subcarriers within the $n$th reported SB. (The exact nature of $\mathbf{x}_{i, n}$ depends on the feedback method adopted.) Furthermore, let $q_{i, \max }\left(\mathbf{x}_{i, n}\right) \in$ $\{1,2, \ldots, J\}$ be the index of the highest-rate MCS that can be supported by user $i$ for the $n$th SB at CQI value $\mathbf{x}_{i, n}$, that is, $q_{i, \max }\left(\mathbf{x}_{i, n}\right)=\arg \max _{j}\left(R_{j}^{(c)} \log _{2}\left(M_{j}\right) \mid \mathbf{x}_{i, n}\right)$. Due to frequency selectivity, the qualities of the subcarriers within a SB may differ; the indicator $\mathbf{x}_{i, n}$ should provide a good collective representation of the qualities for all the subcarriers within the $n$th SB [10-12]. For convenience, we assume that the MCS rate $R_{j}^{(c)} \log _{2}\left(M_{j}\right)$ increases monotonically with $j$, and that the rate of MCS 1 is zero. SBs whose CQI values are not reported back are assigned to MCS 1 .

As mentioned earlier, in a non-MIMO configuration, all SBs scheduled for a given user within the same TTI must use the same MCS. If MCS $j$ is to be used for user $i$, then only certain SBs can be assigned to the user. For example, suppose $N_{i}=4$, and

$$
1 \leq q_{i, \max }\left(\mathbf{x}_{i, 2}\right)<q_{i, \max }\left(\mathbf{x}_{i, 1}\right)<q_{i, \max }\left(\mathbf{x}_{i, 3}\right)<q_{i, \max }\left(\mathbf{x}_{i, 5}\right) \leq J .
$$

Then, if MCS $j=q_{i, \max }\left(\mathbf{x}_{i, 3}\right)$ is used, only SBs $n=3$ and 5 can be allocated to user $i$ since only these SBs have good enough channel qualities to support an MCS index of $q_{i, \max }\left(\mathbf{x}_{i, 3}\right)$ or higher. Selecting SBs $n=1$ or 2 with MCS $j=q_{i, \max }\left(\mathbf{x}_{i, 3}\right)$ would result in unacceptably high error rates for these SBs. On the other hand, if $j=q_{i, \max }\left(\mathbf{x}_{i, 2}\right)$, all 4 SBs can be selected, at the expense of a lower bit rate for SBs 1, 3, and 5. This suggests that there is an optimal value of $j$ which maximizes the total bit rate for user $i$.

\section{Optimal Scheduler}

\subsection{Multiuser Optimization Model}

With multiple users, the optimization problem is more difficult. In addition, each SB can only be occupied by a single user [4]. Let

$$
v_{i, n}\left(\mathbf{x}_{i, n}\right)=\sum_{j=1}^{q_{i, \max }\left(\mathbf{x}_{i, n}\right)} b_{i, j} r_{j}
$$

be the bit rate of $\mathrm{SB} n$ selected for user $i$ given the channel quality $\mathbf{x}_{i, n}$, where $b_{i, j} \in\{0,1\}$ is a binary decision variable. Let $Q_{\text {max }}(i)=\max _{n \in \mathcal{N}_{i}}\left\{q_{i, \max }\left(\mathbf{x}_{i, n}\right)\right\}$. The constraint

$$
\sum_{j=1}^{Q_{\max }(i)} b_{i, j}=1
$$

is introduced to ensure that the MCS for user $i$ can only take on a single value between 1 and $Q_{\max }(i)$. The formulation in (3) allows the selected bit rate for SB $n$ to be less than what $\mathbf{x}_{i, n}$ can potentially support, as may be the case if user $i$ is assigned more than one SB during a TTI. From (3) and (4), it can be seen that SB $n$ might be selected for user $i$ only if the MCS $j^{*}$ chosen for user $i$ satisfies $j^{*} \leq q_{i, \max }\left(\mathbf{x}_{i, n}\right)$.

The problem of jointly maximizing the sum of the bit rates for all users can be formulated as

$$
\text { (P1): } \max _{\mathbf{A}, \mathbf{B}} \sum_{i=1}^{U} \sum_{n \in \mathcal{N}_{i}} a_{i, n} \sum_{j=1}^{q_{i, \max }\left(\mathbf{x}_{i, n}\right)} b_{i, j} r_{j}
$$

subject to (4) and

$$
\sum_{i=1}^{U} a_{i, n}=1, \quad n \in \mathcal{N}_{i}, a_{i, n}, b_{i, j} \in\{0,1\}, \forall i, j, n .
$$

In problem $(\mathrm{P} 1), \mathbf{A}=\left\{a_{i, n}, i=1, \ldots, U, n \in \mathcal{N}_{i}\right\}, \mathbf{B}=$ $\left\{b_{i, j}, i=1, \ldots, U, j=1, \ldots, Q_{\max }(i)\right\}$, and $a_{i, n}$ is a binary decision variable, with value 1 if $\mathrm{SB} n$ is assigned to user $i$ and 0 otherwise. The objective in (5) is to select optimal values for $\mathbf{A}$ and $\mathbf{B}$ to maximize the aggregate bit rate $\sum_{i=1}^{U} \sum_{n \in \mathcal{N}_{i}} a_{i, n} v_{i, n}\left(\mathbf{x}_{i, n}\right)$.

\subsection{Linearized Model}

Note that Problem (P1) is nonlinear due to the product $a_{i, n} b_{i, j}$ in (5). Although solutions can be obtained using optimization techniques such as Branch-and-Bound [13], global optimality cannot be guaranteed. To avoid this difficulty, the problem can be transformed into an equivalent linear problem by introducing an auxiliary variable $t_{n, i, j}=$ $a_{i, n} b_{i, j}$. Then, Problem (P1) can be linearized as follows:

$$
\left(\mathrm{P}^{\prime}\right): \max _{\mathbf{A}, \mathbf{B}, \mathbf{T}} \sum_{i=1}^{U} \sum_{n \in \mathcal{N}_{i}} \sum_{j=1}^{q_{i, \max }\left(\mathbf{x}_{i, n}\right)} t_{n, i, j} r_{j}
$$

subject to (4), (6) and

$$
\begin{aligned}
& t_{n, i, j} \leq b_{i, j}, \\
& t_{n, i, j} \leq a_{i, n} M, \\
& t_{n, i, j} \geq b_{i, j}-\left(1-a_{i, n}\right) M,
\end{aligned}
$$

where $M$ is a large positive real value. Problem $\left(\mathrm{P} 1^{\prime}\right)$ can then be solved using well-known integer linear programming techniques [13]. 


\section{A Suboptimal Scheduler}

In the optimal scheduler formulations in $(\mathrm{P} 1)$ and $\left(\mathrm{P} 1^{\prime}\right)$, the MCSs, SBs, and users are jointly assigned. To reduce complexity, the proposed suboptimal scheduler performs the assignment in two stages. In the first stage, each SB is assigned to the user who can support the highest bit rate. In the second stage, the best MCS for each user is determined. The idea behind the suboptimal scheduler is to assign a disjoint subset of SBs to each user, thereby reducing a joint multiuser optimization problem into $U$ parallel single-user optimization problems.

Let $\varphi_{n}$ be the index of the user which can support the highest-rate MCS for $\mathrm{SB} n$, that is, $\varphi_{n}=$ $\arg \max _{i \in\{1,2, \ldots, U\}} q_{i, \max }\left(\mathbf{x}_{i, n}\right)$. Furthermore, let $\widetilde{\mathcal{N}}_{i}$ be the (disjoint) set of SBs assigned to user $i$, that is, $\left\{n\right.$ such that $\varphi_{n}=$ $i$. In the first stage, the suboptimal scheduler determines $\left\{\widetilde{\mathcal{N}}_{i}\right\}_{i=1}^{U}$.

Let $Q_{\max }^{\prime}(i)=\max _{n \in \widetilde{\mathcal{N}}_{i}}\left\{q_{i, \max }\left(\mathbf{x}_{i, n}\right)\right\}$, and let the MCS vector, $\mathbf{b}_{i}$, for user $i$ be

$$
\mathbf{b}_{i}=\left[b_{i, 1}, b_{i, 2}, \ldots, b_{i, Q_{\max }^{\prime}(i)}\right] .
$$

In the second stage, the suboptimal scheduler determines $\mathbf{b}_{i}$ which maximizes the total bit rate for user $i$. Similar to the approach in Section 3, the optimal $\mathbf{b}_{i}$ can be obtained by solving the following problem:

$$
\text { (P2): } \max _{\mathbf{b}_{i}} \sum_{n \in \widetilde{\mathcal{N}}_{i}} \sum_{j=1}^{q_{i, \max }\left(\mathbf{x}_{i, n}\right)} b_{i, j} r_{j},
$$

s.t.

$$
\sum_{j=1}^{Q_{\text {max }}^{\prime}(i)} b_{i, j}=1, \quad b_{i, j} \in\{0,1\}, \forall i, j .
$$

Compared to (P1) or $\left(\mathrm{P}^{\prime}\right),(\mathrm{P} 2)$ is a much simpler problem.

\section{Numerical Results}

For illustration purposes, we assume $N_{\text {tot }}=12 \mathrm{SBs}$ per TTI, $L=12$ subcarriers per $\mathrm{SB}, N_{1}=N_{2}=\cdots=N_{U}=N$, and that the normal cyclic prefix configuration is used [4]. The fading amplitude for each subcarrier and user follows the Nakagami- $m$ model [14], with a fading figure $m=1$. The average signal-to-interference plus noise ratios (SINRs) for the users are $10 \mathrm{~dB}, 11 \mathrm{~dB}$, and $12 \mathrm{~dB}$, respectively. It is assumed that the SINRs for all subcarriers of each user are correlated, but identically distributed (c.i.d.), and that the resource blocks follow the localized configuration [5]. The correlation coefficient between a pair of subcarriers is given by $\rho^{|i-j|}$, where $i$ and $j$ are the subcarrier indices. The SINR of each subcarrier is assumed to be independent at the beginning of each scheduling period, and constant throughout the entire period. For simplicity, it is assumed that the set of MCSs consists of QPSK $1 / 2$ and 3/4, 16QAM 1/2 and 3/4, as well as 64-QAM 3/4 [1], and the L1/L2 control channels are mapped to the first OFDM symbol

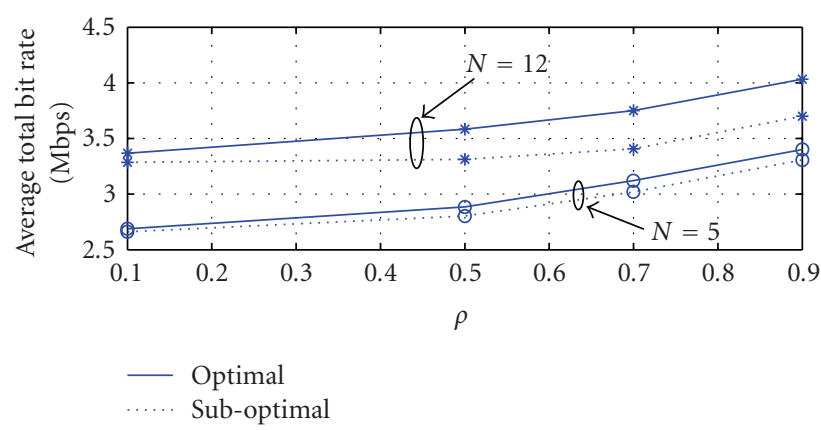

(a)

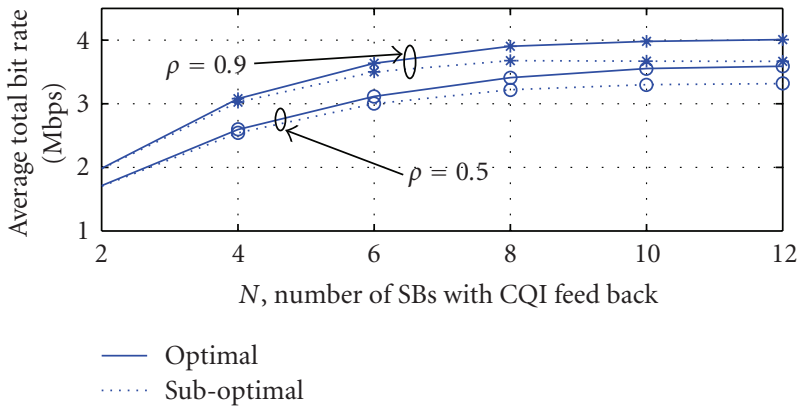

(b)

Figure 1: Average total bit rate as a function of $\rho$ and $N$, respectively, with $U=3$. (a) $N=5$ and 12 with $U=3$. (b) $\rho=0.5$ and 0.9 .

within each subframe. Furthermore, each subframe consists of 8 reference symbols [4]. The feedback method is based on the exponential effective SINR mapping (EESM) [10], with parameter values obtained from [15]. Let $R_{\mathrm{tot}}^{*}$ be the total bit rate defined in (5) or (7), and $E\left[R_{\mathrm{tot}}^{*}\right]$ be the value of $R_{\mathrm{tot}}^{*}$ averaged over 2500 channel realizations.

Figure 1 shows the average total bit rate, $E\left[R_{\mathrm{tot}}^{*}\right]$, as a function of $\rho$ (top) and $N$ (bottom). In Figure 1(a), it can be observed that the performance improves with the level of correlation among subcarriers. Recall that the idea behind EESM is to map a set of subcarrier SINRs, $\left\{\Gamma_{i}\right\}_{i=1}^{L}$, to a single effective SINR, $\Gamma^{*}$, in such a way that the block error probability (BLEP) due to $\left\{\Gamma_{i}\right\}_{i=1}^{L}$ can be well approximated by that at $\Gamma^{*}$ in additive white Gaussian noise (AWGN) $[1,10]$. The value of $\Gamma^{*}$ tends to be skewed towards the weaker subcarriers in order to maintain an acceptable BLEP. At a low value of $\rho$, subcarriers with large SINRs are not effectively utilized, leading to a relatively poor performance. In Figure 1(b), it can be seen that the performance improves with $N$, but the rate of improvement decreases. There is little performance improvement as $N$ increases beyond 8 .

Figure 2(a) shows $E\left[R_{\text {tot }}^{*}\right]$ as a function of the number, $U$, of users for $\rho=0.9$ and $N=12$. The average SINRs for all users are set to $10 \mathrm{~dB}$. As $U$ increases, $E\left[R_{\text {tot }}^{*}\right]$ increases due to the more pronounced benefits from multiuser diversity. Figure 2(b) shows the percentage gain in $E\left[R_{\mathrm{tot}}^{*}\right]$ for the optimal scheduler relative to the suboptimal scheduler as a function of $U$. As $U$ increases, it becomes increasingly likely 


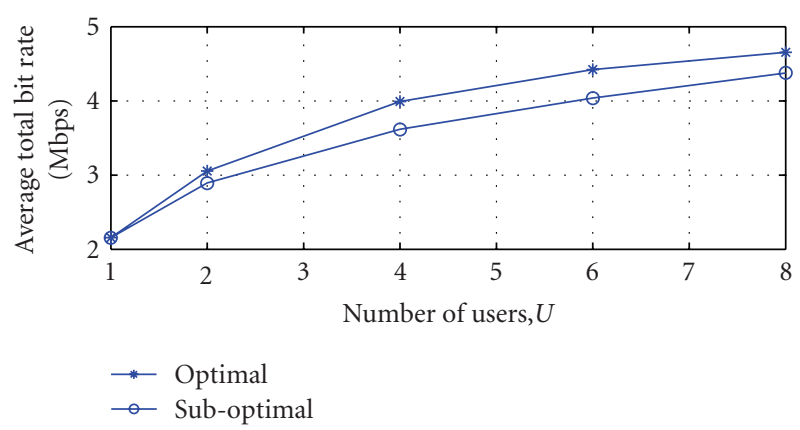

(a)

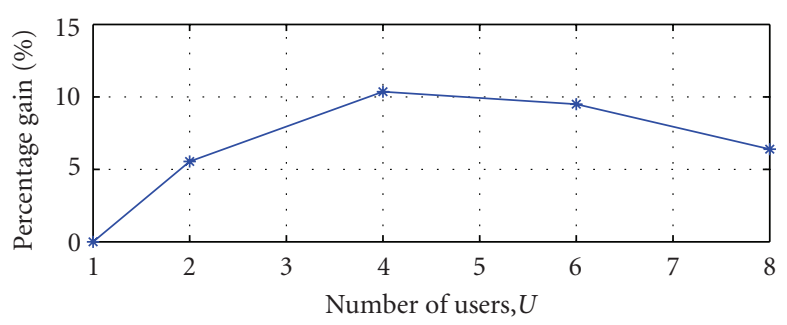

(b)

FIGURE 2: Average total bit rate and percentage gain as a function of the number of users, $U$, with $\rho=0.9$ and $N=12$.

that a given user will be assigned at most one SB in the first stage operation of the suboptimal scheduler. In this event, the suboptimal scheduler is actually optimal. It is therefore expected that the difference in performance between the optimal and suboptimal schedulers will be small when $U$ is large, as illustrated in Figure 2(b). The result indicates that the suboptimal scheduler is especially attractive for large values of $U$ since it provides a significant reduction in complexity and its performance approaches that of the optimal scheduler.

\section{Conclusion}

The problem of multiuser downlink scheduling in an LTE cellular communication system was studied. Numerical results show that both the correlation among subcarriers and the amount of information fed back play important roles in determining the system performance. It was found that limited feedback may be sufficient to achieve a good performance. A reduced complexity suboptimal scheduler was proposed and found to perform quite well relative to the optimal scheduler. The suboptimal scheduler becomes especially attractive as the number of users increases.

\section{Acknowledgments}

This work was supported in part by the Natural Sciences and Engineering Research Council (NSERC) of Canada under Grant no. OGP0001731, by the UBC PMC-Sierra Professorship in Networking and Communications, and by a Marie Curie Post-Doctoral Fellowship.

\section{References}

[1] J. G. Andrews, A. Ghosh, and R. Muhamed, Fundamentals of WiMAX: Understanding Broadband Wireless Networking, Prentice Hall, Upper Saddle River, NJ, USA, 2007.

[2] H. Liu and G. Li, OFDM-Based Broadband Wireless Networks, Design and Optimization, John Wiley \& Sons, New York, NY, USA, 2005.

[3] G. Wunder, C. Zhou, H.-E. Bakker, and S. Kaminski, "Throughput maximization under rate requirements for the OFDMA downlink channel with limited feedback," EURASIP Journal on Wireless Communications and Networking, vol. 2008, Article ID 437921, 14 pages, 2008.

[4] E. Dahlman, S. Parkvall, J. Sköld, and P. Beming, 3 G Evolution: HSPA and LTE for Mobile Broadband, Academic Press, New York, NY, USA, 2007.

[5] "Evolved universal terrestrial radio access (EUTRA); physical channels and modulation (Release 8)," Tech. Rep. 3GPP TS36.211, 3rd Generation Partnership Project, SophiaAntipolis Cedex, France, September 2007.

[6] X. Ning, Z. Ting, W. Ying, and Z. Ping, "A MC-GMR scheduler for shared data channel in 3GPP LTE system," in Proceedings of the 64th IEEE Vehicular Technology Conference (VTC '06), pp. 1-5, Montreal, Canada, September 2006.

[7] K. I. Pedersen, G. Monghal, I. Z. Kovács, et al., "Frequency domain scheduling for OFDMA with limited and noisy channel feedback," in Proceedings of the 66th IEEE Vehicular Technology Conference (VTC '07), pp. 1792-1796, Baltimore, Md, USA, September-October 2007.

[8] S. T. Chung and A. J. Goldsmith, "Degrees of freedom in adaptive modulation: a unified view," IEEE Transactions on Communications, vol. 49, no. 9, pp. 1561-1571, 2001.

[9] N. Miki, Y. Kishiyama, K. Higuchi, and M. Sawahashi, "Optimum adaptive modulation and channel coding scheme for frequency domain channel-dependent scheduling in OFDM based evolved UTRA downlink," in Proceedings of IEEE Wireless Communications and Networking Conference (WCNC '07), pp. 1785-1790, Hong Kong, March 2007.

[10] Ericsson, "System-Level Evaluation of OFDM-Further Considerations," TSG-RAN WG1 \#35, Lisbon, Portugal, TR R1031303, November 2003.

[11] Y. W. Blankenship, P. J. Sartori, B. K. Classon, V. Desai, and K. L. Baum, "Link error prediction methods for multicarrier systems," in Proceedings of the 60th IEEE Vehicular Technology Conference (VTC '04), vol. 6, pp. 4175-4179, Los Angeles, Calif, USA, September 2004.

[12] M. Lampe, T. Giebel, H. Rohling, and W. Zirwas, "PERprediction for PHY mode selection in OFDM communication systems," in Proceedings of IEEE Global Telecommunications Conference (GLOBECOM '03), vol. 1, pp. 25-29, San Francisco, Calif, USA, December 2003.

[13] R. Rardin, Optimization in Operations Research, Prentice Hall, Upper Saddle River, NJ, USA, 1998.

[14] M. K. Simon and M.-S. Alouini, Digital Communication over Fading Channels, John Wiley \& Sons, New York, NY, USA, 2nd edition, 2005.

[15] E. Westman, Calibration and evaluation of the exponential effective SINR mapping (EESM) in 802.16, M.S. thesis, The Royal Institute of Technology (KTH), Stockholm, Sweden, September 2006. 

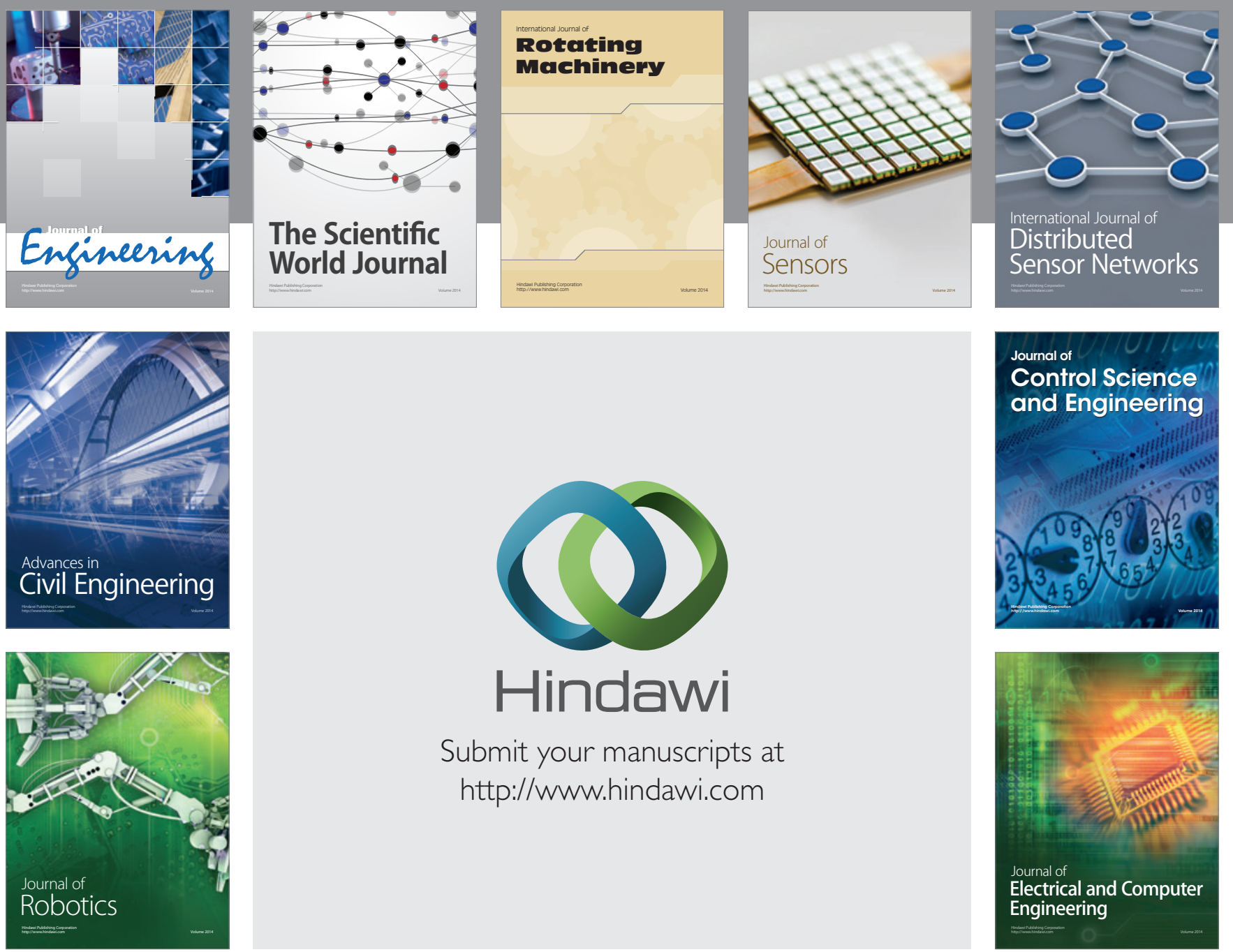

Submit your manuscripts at

http://www.hindawi.com
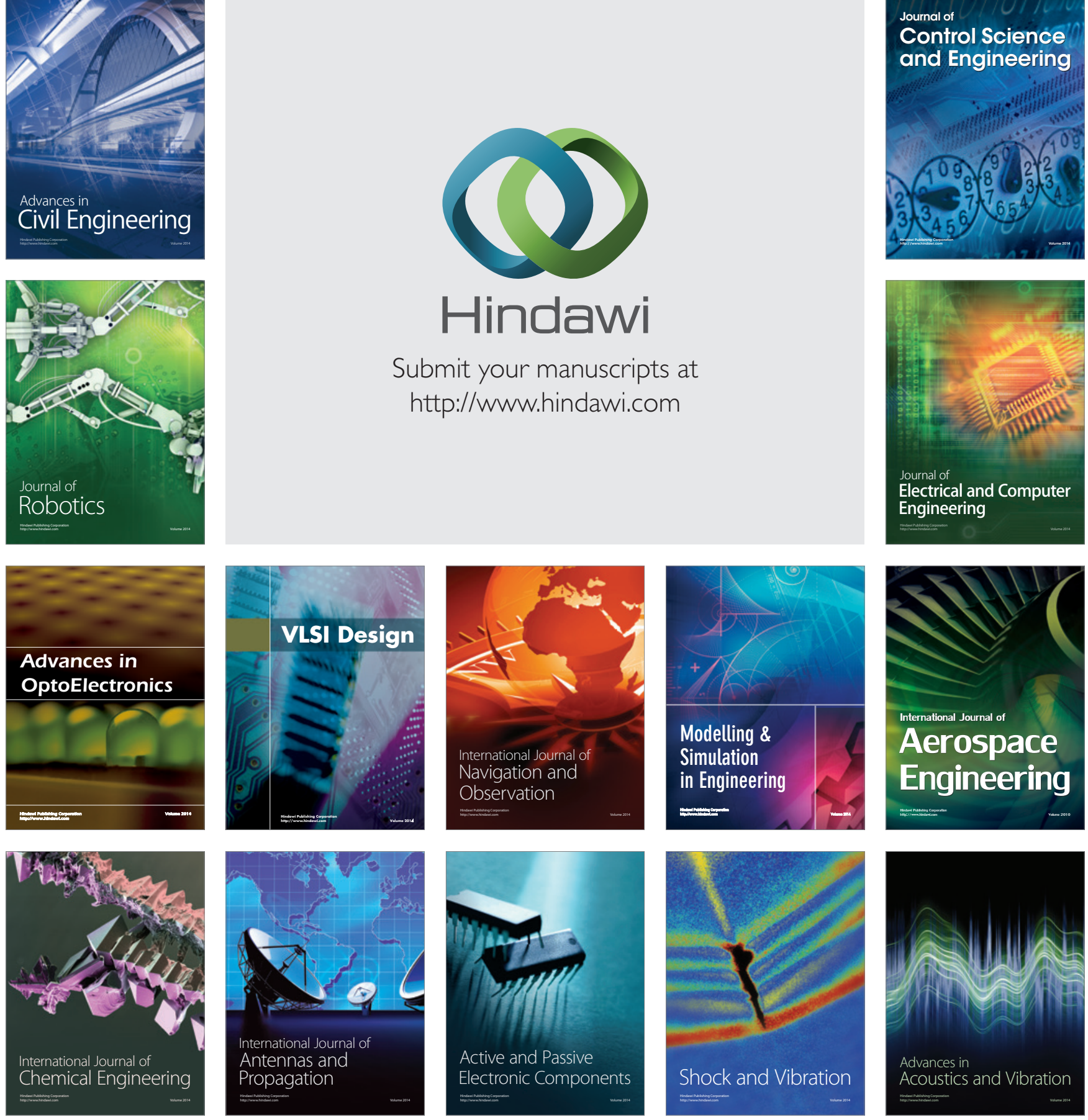